\title{
Penetration Route of the Selective Glucocorticoid Receptor Agonist SA22465 and Betamethasone into Rabbit Meibomian Gland Based on Pharmacokinetics and Autoradiography
}

\author{
Nagayoshi Asano, Kenji Ueda, and Kouichi Kawazu \\ Nara Research and Development Center, Santen Pharmaceutical Co., Ltd., Nara, Japan
}

Received February 16, 2017; accepted April 20, 2017

\begin{abstract}
Meibomian glands are modified sebaceous glands embedded within specific types of dense connective tissues. This study investigated drug penetration into meibomian glands following a single topical administration in rabbits. We measured time course concentrations of the selective glucocorticoid receptor agonist (SEGRA) SA22465 and betamethasone in lid margins, palpebral conjunctival epithelium, and meibomian glands following a single instillation using a newly established collection method. We also visualized the distribution of ${ }^{14} \mathrm{C}$-SA22465 in eyelid tissue sections using microautoradiography. Concentrations of SA22465 and its major metabolite SA22313 were highest in lid margins, followed by palpebral conjunctival epithelium and meibomian glands in a 100-fold descending order. Betamethasone exhibited similar distribution profiles with smaller concentration differences. The distribution of silver grains as
\end{abstract}

a quantitative index of radioactivity in eyes and eyelids was determined in a subjective manner using microautoradiographs, which revealed that the highest distribution of silver grains was associated with the cornea, followed by posterior segment tissues, such as the sclera, choroid, and retina. Low levels were associated with more internal ocular tissues and a greater number of compartments. Moderate levels of radioactivity were associated with meibomian glands and connective tissues, including the nictitating membrane. In contrast, meibomian ducts contained only background levels of radioactivity. Our findings indicate that the transconjunctiva is the most likely route of drug entry into meibomian glands following ocular administration rather than the central meibomian duct; however, this distribution is limited. A physiologic barrier may restrict drug penetration across the tarsal plate.

\section{Introduction}

Meibomian gland dysfunction (MGD) is a major cause of dry eye, having a widespread prevalence worldwide with the incidence increasing with age (Lemp and Nichols, 2009; Schaumberg et al., 2011; Viso et al., 2012; Siak et al., 2012). The current treatment options for MGD are primarily palliative and limited to eyelid hygiene with warm compression and empirical antimicrobial therapy (Geerling et al., 2011). Existing evidence is insufficient to confirm the efficacy of oral antibiotic therapy (Wladis et al., 2016). Alternative options, including intraductal probing (Maskin, 2010), an automated thermodynamic system (Finis et al., 2014), and anti-inflammatory therapy, have emerged more recently (Qiao and Yan, 2013). However, each option has pros and cons, and no approved drug is pharmacologically viable for treating MGD. Therefore, the development of a highly effective drug is a longsought goal for MGD therapy.

MGD is not completely understood; however, several pathogenic mechanisms were proposed based on the clinical symptoms, such as hyperkeratinization of ductal epithelium, bacterial flora changes of the lid margin (Dougherty and McCulley, 1986), lipid profile alterations (Green-Church et al., 2011), and meibomian gland atrophy (Jester et al., 2015) (Baudouin et al., 2016). Such multifactorial mechanisms underlying MGD suggest that a therapeutic drug should be delivered to specific cells in the meibomian glands depending on the expected mode

https://doi.org/10.1124/dmd.117.075473. of action. However, very little is known regarding the mechanism of drug uptake into the meibomian glands.

Meibomian glands are modified, holocrine, sebaceous glands that are surrounded by connective tissue called the tarsal plate, which maintains the physical structure of the eyelids. The tarsal plate is composed of a variety of extracellular matrix components, such as collagens, proteoglycans, and glycosaminoglycans (Milz et al., 2005). It exhibits mixed features of dense fibrous connective tissue and hyaline cartilage. In general, a collagen- and elastin-enmeshed fibril network as well as cartilage constitute a diffusion barrier, especially for certain compounds with a high molecular size or fixed negative charge, which prevents accessibility to target tissues (Maroudas, 1976). Although the meibomian gland itself is encapsulated in a distinctive "territorial" matrix, a drug may reach the meibomian glands via the central meibomian duct. The central duct plays a physiologically important role as the excretory route for meibomian oil (meibum) toward the orifice to maintain ocular surface homeostasis (Bron et al., 2004); however, whether a drug can proceed against the driving force of meibum secretion toward the lid margin remains controversial.

Human pharmacokinetic studies are challenging, particularly considering the meibomian gland, from both ethical and cosmetic perspectives. A different technical hurdle must also be overcome in animal studies. Meibomian glands lie just behind the eyelash lining along the eyelid margin, and due to their unique location and structure, no satisfactory meibomian gland collection method has been established. Several extraction approaches have been evaluated; however, these techniques

ABBREVIATIONS: SEGRA, a selective glucocorticoid receptor agonist; MGD, meibomian gland dysfunction; HPLC, high-performance liquid chromatography; LC-MS/MS, liquid chromatography-tandem mass spectrometry. 
were not optimal for the desired throughput for typically time-consuming and labor-intensive pharmacokinetic studies (e.g., freeze dissections and temperature-dependent enzymes) (Maskin and Tseng, 1991). Most importantly, meibomian glands must be collected without contamination from highly exposed ocular surface tissues or drug particles. This indicates that a robust meibomian gland collection methodology is required to advance our understanding of the complex biodistribution in meibomian glands and improve drug screening for MGD.

The etiology of MGD is uncertain, but inflammation of the posterior lid margin is one of the apparent clinical signs. Indeed, topical steroids are frequently used to treat MGD in combination with antimacrobiotics, expecting to show potent anti-inflammatory efficacy via glucocorticoid receptor binding on meibomian gland epithelial cells; however, despite its powerful therapeutic actions, these agents are only used in the short term because of potential adverse events. SEGRA is believed to be dissociated from steroid-related adverse events, especially intraocular pressure elevation, which creates a desirable option for the treatment of ocular inflammatory disorders, including MGD. Hence, the characterization of the pharmacokinetic profile of steroids in the meibomian glands is an informative benchmark in drug screening for MGD.

This study first developed and validated a meibomian gland collection procedure that is rapid, precise, and reproducible. We used this streamlined new method to determine the concentrations of betamethasone sodium phosphate (a water-soluble corticosteroid) and the selective glucocorticoid receptor agonist SA22465 (a poorly soluble molecule administered as a suspension) in the lid margins and palpebral conjunctival epithelium and meibomian glands in rabbits, considering any notable differences in penetration between aqueous solution and suspension. We also visualized the distribution of ${ }^{14} \mathrm{C}-\mathrm{SA} 22465$ within eyelid tissue sections using macro- and microautoradiography to ascertain the degree of penetration of SA22465 into meibomian glands.

\section{Material and Methods}

Chemicals. SA22465, its metabolite SA22313, and the internal standard (free form)-d3 were synthesized in our laboratories. ${ }^{14} \mathrm{C}-\mathrm{SA} 22465(2.15 \mathrm{MBq} / \mathrm{mg})$ was synthesized by Sekisui Medical (Ibaraki, Japan). Radiochemical purity was determined to be $99.6 \%$ using high-performance liquid chromatography (HPLC). Betamethasone sodium phosphate and prednisolone were purchased from SigmaAldrich (St. Louis, MO). Betamethasone sodium phosphate was dissolved in saline for ophthalmic formulation. In contrast, SA22465 was suspended with vehicle for ophthalmic formulation due to poor water solubility below $100 \mathrm{ng} / \mathrm{ml}$. The assay of SA22465 ophthalmic suspension was determined to be $97.6 \%$ by an external standard method. SA22313 impurity level was calculated as $0.16 \%$ in relation to total area of peaks. The SA22465 vehicle contained sodium dihydrogen phosphate as the buffering agent, polysorbate 80 as an suspension stabilizer, glycerin as an isotonic agent, sodium hydroxide as a pH-adjusting agent (targeting $\mathrm{pH} 7$ ), and benzalkonium chloride as a preservative. The average particle size of SA22465 ophthalmic suspension was measured to be $2.9 \mu \mathrm{m}$. The binding affinities of SA22465, SA22313, and betamethasone sodium phosphate to human glucocorticoid receptor $\left(\mathrm{IC}_{50}\right.$ value) were $23.6,116.4$, and $67.1 \mathrm{ng} / \mathrm{ml}$, respectively. All other reagents and solvents were commercial products of analytical grade.

Animals. Male Japanese white rabbits were used for pharmacokinetic studies (body weight: 1.6-2.1 kg). The rabbits were individually housed in cages in an airconditioned room and maintained on a standard laboratory diet (LRC4; Oriental Yeast, Tokyo, Japan). Male New Zealand white rabbits were used for autoradiography (body weight: $2.3-2.5 \mathrm{~kg}$ ). These rabbits were also singly housed and fed a standard diet (Teklad Global Rabbit Diet 2030; Harlan Laboratories, Blackthorn, Oxfordshire, UK). All animals had free access to food and water and were maintained under a 12:12-hour light/dark cycle. All animal care and experimental procedures were performed in an accredited laboratory according to international guidelines as well as in compliance with the Ethical Guidelines for Animal Experiments of Santen Pharmaceutical.

Meibomian Gland Collection. Rabbits were euthanized with an overdose of pentobarbital. Upper eyelids were excised and rinsed with saline. Epithelial cells were scraped from palpebral conjunctiva using a golf knife (MGL24; MANI, Tochigi, Japan), and the eyelids were gently placed onto microscope slides with the skin side facing up. The slides were placed on a dry ice block throughout the procedure. The eyelids froze on contact and stuck tightly to the slides due to the ice crystal lattice formed between the surface of tissue and the slides. The eyelid skin and orbicularis muscle were gently removed via manually slicing with a microtome blade (A35; FEATHER Safety Razor, Osaka, Japan) until small blood vessels (i.e., the peripheral tarsal arcade) became visible at the center of each eyelid. Meibomian gland orifices were removed with the eyelashes to minimize potential contamination. Extra connective tissues were also removed, and the meibomian glands within each tarsal plate (approximately $10 \mathrm{mg}$ ) were collected in multiple thin-sliced sections. Collected meibomian glands were immediately frozen and stored at $-80^{\circ} \mathrm{C}$ until subsequent liquid chromatography-tandem mass spectrometry (LC-MS/MS) analyses.

Pharmacokinetic Study. An aliquot of $50 \mu \mathrm{l}$ of a 1\% SA22465 ophthalmic suspension or $1 \%$ betamethasone sodium phosphate solution was instilled into both eyes of albino rabbits $(0.5 \mathrm{mg} / \mathrm{eye})$. The rabbits were euthanized using an overdose of intravenous pentobarbital at specified time points. The surfaces of the eyes and conjunctiva were rinsed with saline to remove drug particles, and the upper eyelids were surgically excised. The lid margins, conjunctival epithelium, and meibomian glands were collected as described above.

LC-MS/MS Analysis for SA22465 and SA22313. Concentrations of SA22465 and SA22313 were determined using an LC-MS/MS method that was developed and validated in our laboratories. Briefly, tissues were homogenized with stainless steel balls ( $5 \mathrm{~mm}$ diameter) in $250 \mu \mathrm{l}$ of $10 \%$ formic acid/acetonitrile $(33: 67, \mathrm{v} / \mathrm{v})$ for 10 minutes and centrifuged $\left(11,000 \mathrm{~g}, 10\right.$ minutes, $\left.4^{\circ} \mathrm{C}\right)$. Supernatants were recovered, and the samples were diluted $1: 10$ with $2 \%$ formic acid and loaded onto solid-phase extraction plates (OASIS MCX $\mu$ Elution 96-well plate; Waters, Milford, MA). The plates were washed sequentially with $2 \%$ formic acid and acetonitrile, and SA22465 and SA22313 were eluted with $1 \%$ ammonium acetonitrile. The eluent was diluted $1: 1$ with $2 \%$ formic acid and directly injected into a reverse-phase HPLC column (TSK-GEL ODS-100V, $3 \mu \mathrm{m}, 2.0 \mathrm{~mm}$ i.d. $\times$ $50 \mathrm{~mm}$; TOSOH Bioscience, Tokyo, Japan). The mobile phase consisting of $0.1 \%$ formic acid as solvent $\mathrm{A}$ and $0.1 \%$ formic acid acetonitrile as solvent $\mathrm{B}$ was run at a flow rate of $0.5 \mathrm{ml} / \mathrm{min}$ using a gradient program (45-98\% solvent B for 2 minutes, maintained at $98 \%$ solvent B for a further 2 minutes). LC-MS/MS analysis was performed on an Agilent 1100 HPLC system (Agilent Technologies, Santa Clara, CA) coupled with a triple-quadrupole mass spectrometer (API4000; AB Sciex, Foster City, CA) with an electrospray ionization interface. Parameters of the ionization and detection were adjusted with synthetic standards to achieve the highest possible sensitivity. All instrument controls and data processing were performed using Analyst Software version 1.4.2 (AB Sciex).

LC-MS/MS Analysis for Betamethasone. Concentrations of betamethasone were determined using an LC-MS/MS method that was developed and validated in our laboratories. Briefly, samples were homogenized with stainless steel balls ( $5 \mathrm{~mm}$ diameter) for 10 minutes in $250 \mu \mathrm{l}$ of $10 \mathrm{mM}$ ammonium formate. Equal amounts of $10 \mathrm{mM}$ ammonium formate and $500 \mu \mathrm{l}$ of acetonitrile were added. The resulting mixtures were vortexed and centrifuged $(11,000 \mathrm{~g}, 10$ minutes, $\left.4^{\circ} \mathrm{C}\right)$. Supernatants were recovered, and an aliquot was further mixed with $1 \mathrm{ml}$ of t-butyl methyl ether and subsequently centrifuged $\left(1,500 \mathrm{~g}, 10\right.$ minutes, $\left.4^{\circ} \mathrm{C}\right)$. The organic layers were separated and dried up in vacuo at $40^{\circ} \mathrm{C}$. The residues were reconstituted with $150 \mu \mathrm{l}$ of mobile phase [10 $\mathrm{mM}$ ammonium formate/ $0.1 \%$ formic acid/acetonitrile (20:20:60, v/v/v)], and $10 \mu \mathrm{l}$ of an aliquot was injected into a reverse-phase HPLC column (Xterra MS C18, $5 \mu \mathrm{m}, 2.1 \mathrm{~mm}$ i.d. $\times$ $150 \mathrm{~mm}$; Waters). Analytical separation was achieved in an isocratic mode at a flow rate of $0.2 \mathrm{ml} / \mathrm{min}$ within a 5 -minute run.

${ }^{14}$ C-SA22465 Dosing. Eight male rabbits received a single 50- $\mu$ l dose of 3\% ${ }^{14} \mathrm{C}-\mathrm{SA} 22465$ ophthalmic suspension into each eye. The target dose level was $1.5 \mathrm{mg} /$ eye, and the radioactive dose approximately $3.225 \mathrm{MBq}(87 \mu \mathrm{Ci}) / \mathrm{eye}$. ${ }^{14} \mathrm{C}-\mathrm{SA} 22465$ ophthalmic suspension was formulated using the same vehicle as cold material suspension. Duplicate animals were sampled at 1-, 4-, 8-, and 24-hour postdose. Animals were euthanized via an intravenous pentobarbital overdose followed by exsanguination. Once death was confirmed, the eyes and upper and lower eyelids were harvested. These samples were rinsed with saline to remove drug particles from the surface of the tissues and processed for macro- or microautoradiography, as detailed below.

Macroautoradiography. Eyeballs and the associated eyelids were frozen in a hexane/dry ice bath and set in blocks of aqueous $2 \%$ carboxymethylcellulose. 


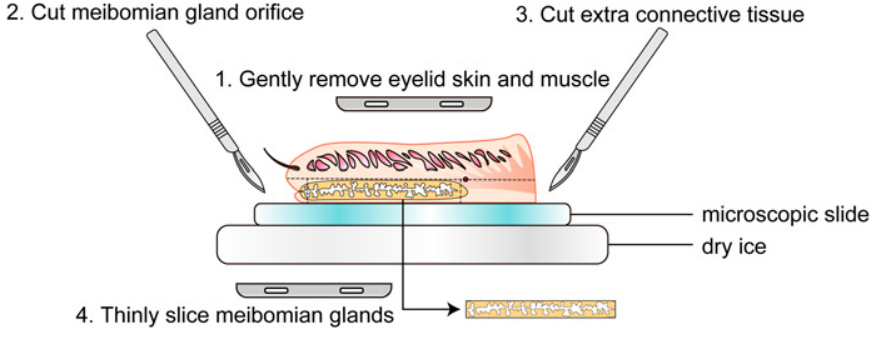

Fig. 1. Schematic illustration of the meibomian gland collection method. Rabbit upper eyelids were placed onto chilled glass slides maintained on dry ice. Eyelid skin was gently removed via manual slicing with a microtome blade until the peripheral tarsal arcade became visible. Meibomian gland orifices and extra connective tissues were removed to avoid potential contamination, and the meibomian glands within the tarsal plate were collected as multiple thin tissue slices.

Numerous cryosections ( $30 \mu \mathrm{m}$ thickness) were taken from the eyes and eyelids at three levels (temporal, midsagittal, and nasal) and collected on an adhesive tape (Filmolux 610 Tape; Neschen, UK) using a Leica CM3600 cryomicrotome maintained at $-20^{\circ} \mathrm{C}$ (Leica Microsystems, Milton Keynes, UK). The sections were freeze-dried and placed in contact with FUJI imaging plates (type BAS-MS; Raytek Scientific Ltd., Sheffield, UK). Blood standards of appropriate activity were also placed in contact with all imaging plates. After exposure in a copperlined, lead chamber for 7 days, the plates were processed at a $100-\mu \mathrm{m}$ resolution using a FUJI FLA-5000 radioluminography system (Raytek Scientific Ltd.). Electronic images were analyzed using a personal computer-based image analysis package (Seescan 2 software, LabLogic Ltd., Sheffield, UK). Tissue concentrations were obtained from tissues and fluids that were visually identified on the autoradiograph by interpolation from each standard curve as dpm per gram of tissue (dpm/g).

Light Microscopic Autoradiography. The remaining eyeballs and eyelids were immersed in precooled optimum cutting temperature compound in molds and snap frozen in a shallow bath of hexane/dry ice. Optimum cutting temperature compound blocks containing the ocular samples were mounted in a Leica CM3050 cryomicrotome maintained at $-12^{\circ} \mathrm{C}$ (Leica Microsystems) and serially sectioned ( $5 \mu \mathrm{m}$ thickness). The semithin sections were thaw-mounted onto microscopic slides precoated by dipping in Ilford $\mathrm{K} 5{ }^{14} \mathrm{C}$ nuclear emulsion (Agar Scientific, Stansted, UK) diluted $1: 1$ with deionized water at $43^{\circ} \mathrm{C}$. The sections were exposed in light-tight cassettes below $-10^{\circ} \mathrm{C}$ for 28 days under safelight conditions and photographically developed for 4 minutes using a Kodak D19 Developer (Eastman Kodak). The slides were stained with hematoxylin and eosin and mounted for microscopic examination.

\section{Results}

Pharmacokinetics of SA22465 in Eyelids after Ocular Instillation in Rabbits. Our pharmacokinetic study was designed to increase throughput and therefore developed rapid, precise, and reproducible manual procedures without specialized instruments that were especially suitable for meibomian gland collection (Fig. 1). The ocular pharmacokinetics of SA22465 and SA22313 (Fig. 2A) were investigated in rabbits following a single ocular administration of a $1 \%$ SA22465 ophthalmic suspension using the newly established method. Figure 3 shows the time-concentration profiles of SA22465 and SA22313 in the lid margin, palpebral conjunctival epithelium, and meibomian glands. It should be noted that SA22313 concentrations may partially represent the delivery from SA22465 suspension containing $0.16 \%$ impurity. SA22465 and SA22313 concentrations were the highest in the lid margin, followed by the palpebral conjunctival epithelium and meibomian glands in a 100fold descending order. SA22465 concentrations were approximately 200-fold higher than SA22313 concentrations in the lid margins at all time points. SA22465 reached its peak concentration of $119,318 \mathrm{ng} / \mathrm{g}$ of tissue at 5 minutes. However, no obvious elimination phase was observed. SA22313 was detectable in lid margins, reached a peak of $534 \mathrm{ng} / \mathrm{g}$ of tissue at the first sampling point (5 minutes) and maintained high concentrations until 24 hours. The peak SA22465 concentration of $12,711 \mathrm{ng} / \mathrm{g}$ of tissue was achieved at 4 hours in the conjunctival epithelium. However, no absorption phase was observed. SA22313 concentrations in the conjunctival epithelium exhibited similar profiles to the lid margin and peaked at the first sampling point (5 minutes) but steeply declined until 30 minutes. Relatively high levels were maintained thereafter. The SA22313 concentration in the meibomian glands was below the lower limit of detection at all time points. Conversely, SA22465 was detectable and reached a $C_{\max }$ of $171 \mathrm{ng} / \mathrm{g}$ of tissue at 30 minutes after instillation; however, there was no obvious elimination phase.

Pharmacokinetics of Betamethasone in Eyelids after Ocular Instillation in Rabbits. Betamethasone sodium phosphate is rapidly converted into its corresponding alcohol in vivo (Samtani et al., 2005). The ocular pharmacokinetics of betamethasone was investigated in rabbits following a single instillation of $1 \%$ betamethasone sodium phosphate solution. Figure 4 shows the time-concentration curves of betamethasone in the lid margin, palpebral conjunctival epithelium, and meibomian glands. The concentration of betamethasone in the conjunctival epithelium was $37,268 \mathrm{ng} / \mathrm{g}$ tissue at 5 minutes. This concentration rapidly declined to $740 \mathrm{ng} / \mathrm{g}$ tissue at 30 minutes and exhibited elimination with a half-life of 2.1 hours. The betamethasone exposure in the lid margin was relatively high, with a peak of $3,810 \mathrm{ng} / \mathrm{g}$ tissue at 1 hour and a gradual decrease over time. The concentration of betamethasone in meibomian glands was approximately 4- to 16-fold lower than that detected in other tissues, with a peak of $189 \mathrm{ng} / \mathrm{g}$ of tissue at 1 hour and moderate elimination with a half-life of 2.3 hours.

Distribution of Radioactivity Using Macroautoradiography. The distribution of radioactivity in the eyeball and eyelids was determined following the ocular administration of ${ }^{14} \mathrm{C}$-SA22465 (Fig. 2B) to rabbits at a target dose level of $1.5 \mathrm{mg} /$ eye. Radioactivity concentrations in various regions of these tissues were assayed using quantitative autoradiography. The results are expressed in nanogram equivalents of SA22465/g of tissue and presented in Table 1 as well as graphically in Fig. 5. The lower limit of quantification for all measurements was $9 \mathrm{ng}$ equivalents of SA22465/g of tissue. Radioactivity was present in all ocular tissues examined at each sampling time. The pattern of distribution at all sampling times was comparable with the highest
A

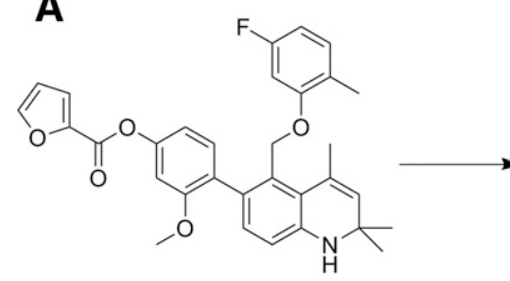

SA22465

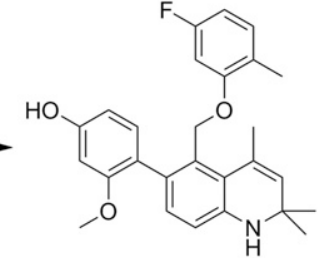

SA22313

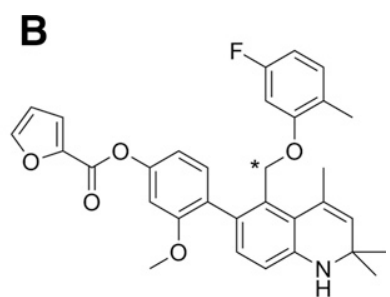

${ }^{14} \mathrm{C}-\mathrm{SA} 22465$
Fig. 2. Chemical structure of SA22465 and SA22313. (A) SA22465 (MW 527.6) is the selective glucocorticoid receptor agonist and metabolized into SA22313 (MW 433.5). (B) ${ }^{14} \mathrm{C}$-SA22465, * signifies the position of radiolabel. 


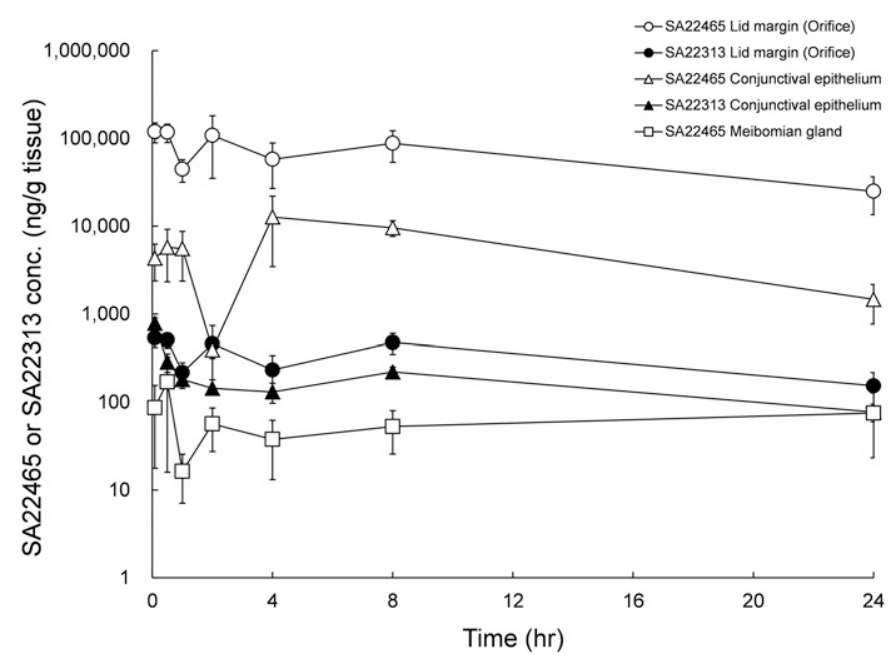

Fig. 3. Lid margin, conjunctival epithelium, and meibomian gland concentration of SA22465 and SA22313 following a single instillation of a 1\% SA22465 ophthalmic suspension to rabbits $(0.5 \mathrm{mg} / 50 \mu \mathrm{l} / \mathrm{eye})$. Values represent the means \pm S.E. ( $n=6$ eyes per group). SA22313 concentrations in meibomian glands were below the limit of quantification at all time points.

concentrations in all animals and associated with the cornea (range: 590 to $176 \mathrm{ng} \mathrm{eq} / \mathrm{g}$ ). Lower quantifiable levels (range: 278 to $10 \mathrm{ng} \mathrm{eq} / \mathrm{g}$ ) were present in the choroid, sclera, retina, and optic nerve, but the concentrations in these structures were below the limit of quantification ( $9 \mathrm{ng} \mathrm{eq} / \mathrm{g}$ ) at some sampling times. Structures deeper in the eyeball, i.e., the lens, pupil, ciliary body, iris, and aqueous humor, contained lower levels of radioactivity (range: 18 to $10 \mathrm{ng}$ eq/g), and radioactivity was not quantifiable in these structures at some sampling times. Radioactivity was below the limit of quantification in the vitreous humor of all animals. There were differences in the tissue concentrations between the duplicate animals at each sampling time, but the general distribution was comparable. The radioactivity associated with the upper and lower eyelids from each animal was also investigated (range: 32,928 to $192 \mathrm{ng}$ $\mathrm{eq} / \mathrm{g}$ ). This radioactivity was primarily associated with the surface of the tissues. Drug and any metabolite molecules associated with the meibomian glands and skin could not be identified or precisely quantified on the autoradiograms because of a halo associated with the

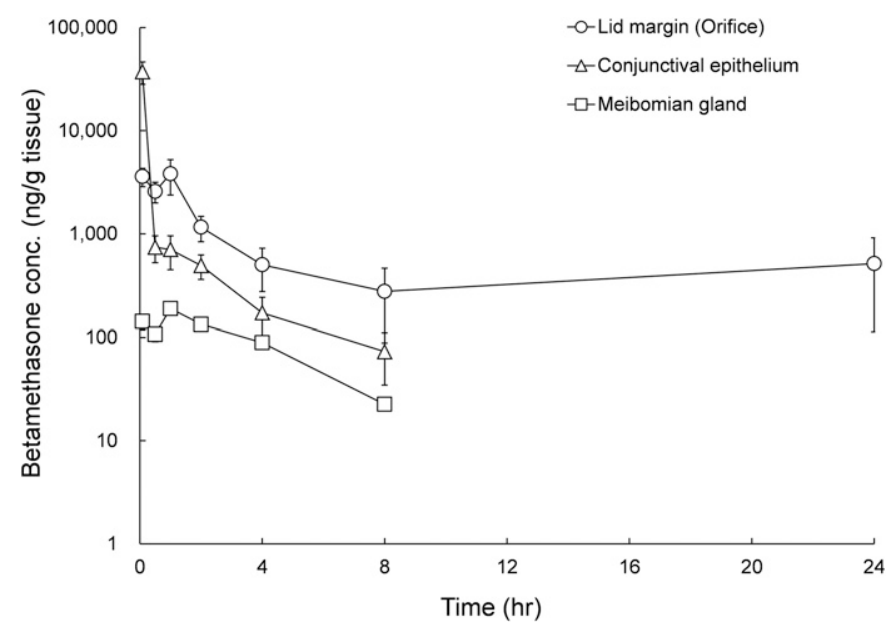

Fig. 4. Lid margin, conjunctival epithelium, and meibomian gland concentration of betamethasone following a single instillation of a $1 \%$ betamethasone sodium phosphate solution to rabbits $(0.5 \mathrm{mg} / 50 \mu \mathrm{l} / \mathrm{eye})$. Values represent the means $\pm \mathrm{S}$.E. ( $n=6$ eyes per group). extremely high levels of radioactivity at the surface of the eyelids, which was caused by a diffusion of radioactivity.

Distribution of Radioactivity Using Microautoradiography. The distribution of radioactivity (silver grains) in the eyeball and eyelids of rabbits was determined in a subjective manner following the ocular administration of ${ }^{14} \mathrm{C}$-SA22465 using microautoradiography techniques. Tissues were harvested at 1-, 4-, 8-, and 24-hours postdose. Positive control slides confirmed that the photography technique worked correctly; i.e., slides flashed to white light were completely fogged (blackened) after processing. The list of tissues that were selected for analysis is presented in Table 2 and graphically illustrated in Fig. 6. The highest distribution of silver grains in the eyeballs was associated with the cornea at all sampling times. A notable pattern was also observed in the other regions examined, namely, moderate to high levels of radioactivity with an increase over time, and this pattern was associated with the structures forming the outer wall of the eyeball (such as the sclera, choroid, and retina) and optic nerve. The ciliary body and iris also contained moderate to high levels of radioactivity. Low levels of detection were associated with the lens and anterior and posterior aqueous and vitreous compartments. Examination of the micrographs for eyelids enabled the identification of several tissues. Areas with very high silver grain density were associated with the surface of the eyelid. These levels were likely related to the presence of the formulated material. The highest levels of radioactivity (ranging from moderate to high) were associated with the hair follicles and hair shafts, with slightly lower levels present around the nictitating membrane in the upper eyelid. There were moderate levels of radioactivity associated with meibomian glands; however, the meibomian ducts contained only low or background levels of radioactivity. Duplicate slides per treatment group were processed, and correlation between tissues from duplicate animals was generally good.

\section{Discussion}

The current study developed an experimental method to characterize the pharmacokinetic profile in meibomian glands based on an improved tissue collection protocol. We previously faced difficulty in generating reliable concentration profiles in meibomian glands because the concentrations of the administered agents measured in meibomian glands widely fluctuated after the eyelid tissues were surgically separated. This resulted in no quantitative difference between the meibomian glands and adjacent tissues such as the conjunctiva (data not shown). The newly established method produced low standard errors when calculating drug concentration and subsequently enabled the time-concentration curve in meibomian glands to shift downward. These consequences were acceptable because our samples contained posterior meibomian glands only to prevent potential contamination. Two distinctive improvements should be highlighted. The procedure was completed within 10 minutes, and special instrumentation was not required. The new method enabled an in-depth pharmacokinetic analysis of eyelid tissues. In the present study, the lid margin exhibited the highest concentrations of SA22465 and betamethasone, as expected. These results may be explained in terms of the drop volume and blinking. The standard instillation volume of ophthalmic drops ranged from 25 to $70 \mu \mathrm{l}$, and pharmacokinetic studies previously demonstrated that the maximal tear film concentration was achieved using a 20- $\mu$ l drop (Nagataki and Mishima, 1980). Increasing drop size beyond this volume does not always increase efficacy but instead can lead to medication waste, especially as a consequence of blinking, forcing some of the therapeutic agent out of the eye (Maurice, 1980). This study applied $50 \mu \mathrm{l}$ of an ophthalmic solution, the ocular surface initially retaining that volume because of surface tension. However, the overflow may lead to wider and more direct exposure to 
TABLE 1

Concentrations of radioactivity in the eyeballs and eyelids of male albino rabbits after ocular administration of ${ }^{14} \mathrm{C}-\mathrm{SA} 22465$ at a nominal dose level of $1.5 \mathrm{mg}$ /eye (macroautoradiography)

\begin{tabular}{|c|c|c|c|c|c|c|c|c|}
\hline \multirow[b]{2}{*}{ Animal number and sex } & \multicolumn{8}{|c|}{ Nanograms Equivalents of SA22465/g of Tissue } \\
\hline & $1, \mathrm{M}$ & $2, \mathrm{M}$ & $3, \mathrm{M}$ & $4, \mathrm{M}$ & $5, \mathrm{M}$ & $6, \mathrm{M}$ & $7, \mathrm{M}$ & $8, \mathrm{M}$ \\
\hline Tissue Sampling time & \multicolumn{2}{|c|}{1 hour } & \multicolumn{2}{|c|}{4 hours } & \multicolumn{2}{|c|}{8 hours } & \multicolumn{2}{|c|}{24 hours } \\
\hline Aqueous humor & 15 & 10 & 11 & BLQ & BLQ & BLQ & BLQ & BLQ \\
\hline Choroid & 38 & 18 & 23 & 12 & 13 & 43 & 19 & BLQ \\
\hline Ciliary body & BLQ & BLQ & BLQ & BLQ & BLQ & BLQ & BLQ & BLQ \\
\hline Cornea & 362 & 294 & 243 & 205 & 213 & 590 & 406 & 176 \\
\hline Iris & BLQ & BLQ & 10 & BLQ & BLQ & BLQ & 12 & BLQ \\
\hline Lens & 13 & BLQ & BLQ & BLQ & BLQ & BLQ & BLQ & BLQ \\
\hline Optic nerve & 87 & 278 & 10 & 14 & 57 & 156 & BLQ & 231 \\
\hline Pupil & 11 & BLQ & BLQ & BLQ & 18 & BLQ & BLQ & BLQ \\
\hline Retina & 37 & BLQ & 18 & 15 & 20 & 18 & BLQ & 20 \\
\hline Sclera & 93 & BLQ & 20 & 15 & 20 & 50 & BLQ & 21 \\
\hline Vitreous humor & BLQ & BLQ & BLQ & BLQ & BLQ & BLQ & BLQ & BLQ \\
\hline Lower eyelid & 32,928 & 3609 & 9290 & 12,575 & 33,673 & 5607 & 15,330 & 27,629 \\
\hline Meibomian gland & ND & ND & ND & ND & ND & ND & ND & ND \\
\hline Skin & ND & ND & ND & ND & ND & ND & ND & ND \\
\hline Upper eyelid & 7,033 & 2,655 & 462 & 3,967 & 192 & 1,084 & 351 & 952 \\
\hline
\end{tabular}

BLQ, tissue concentration below lower limit of quantification; ND, not discernible.

Upper limit of quantification: $36,040 \mathrm{ng}$ equiv/g for all measurements

Lower limit of quantification: $9 \mathrm{ng}$ equiv/g for all measurements

lid margins during blinking. The upper areas near the eyelashes likely maintain higher levels for longer periods, even to the point where drugs may eventually form crystals because tears do not effectively lubricate these sites. This scenario is compatible with our data that betamethasone did not completely disappear from eyelid margins after 8 hours postdose, despite other tissues showing an elimination half-life of approximately

Time after instillation

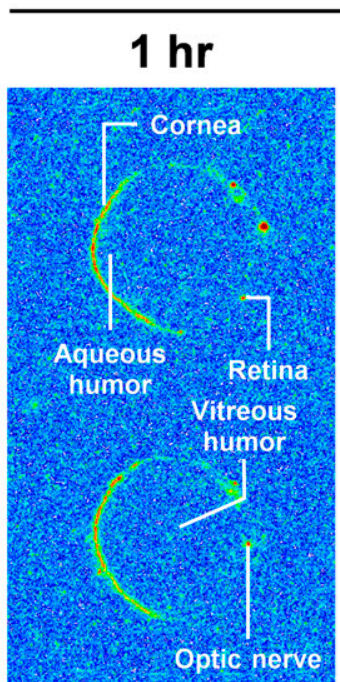

$4 \mathrm{hr}$
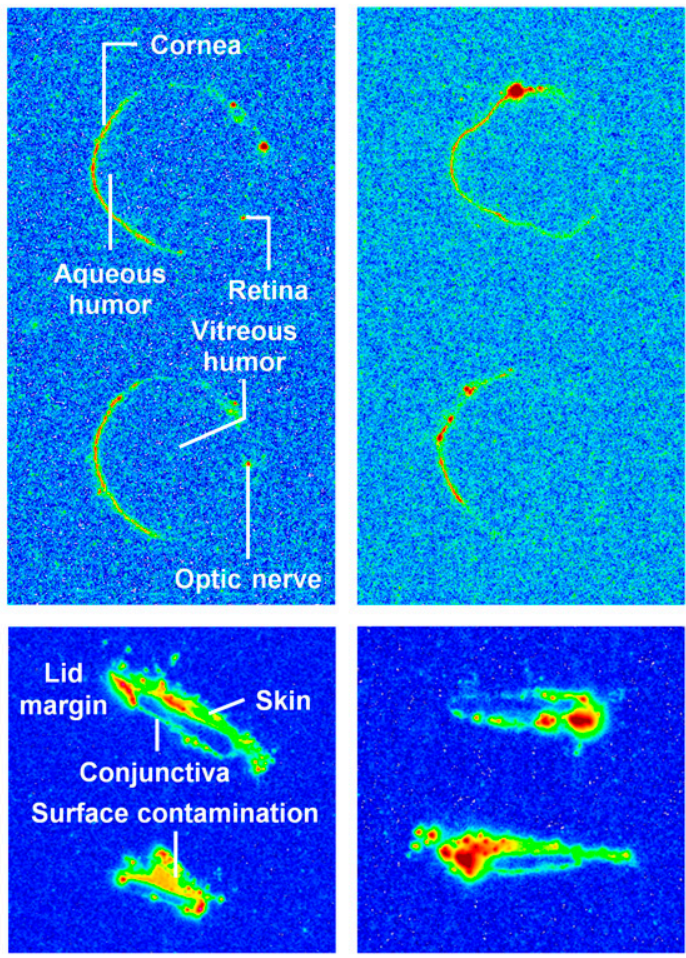

$8 \mathrm{hr}$
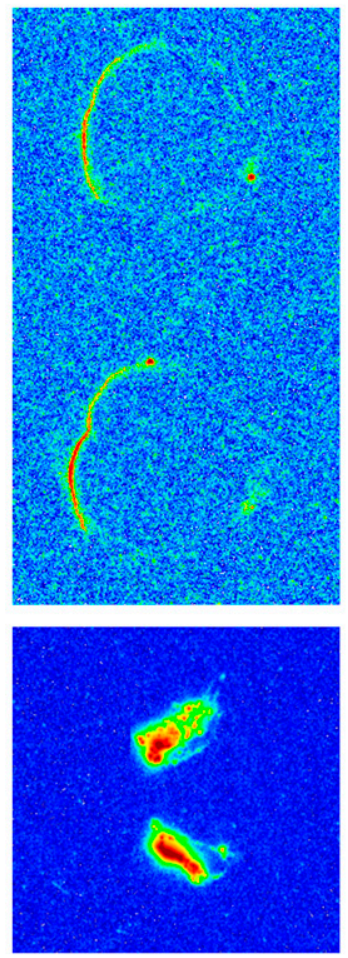

$24 \mathrm{hr}$
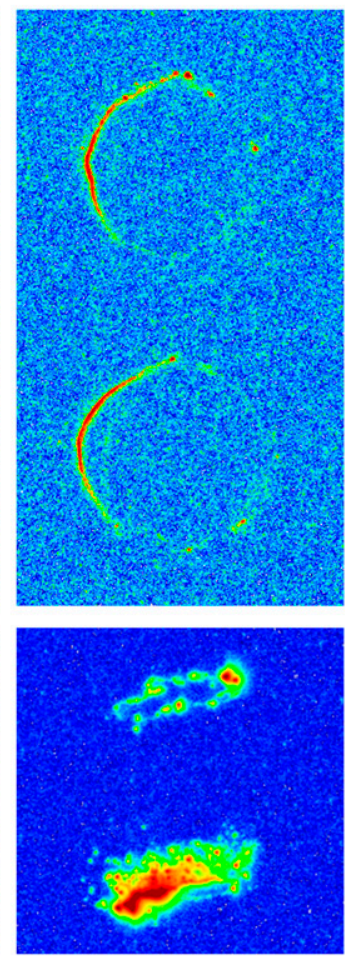

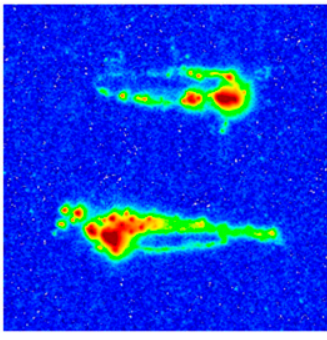

High

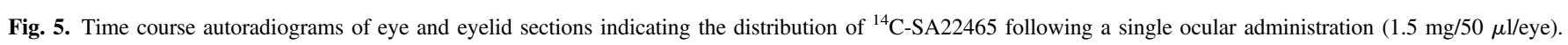


TABLE 2

Qualitative assessment of radioactivity in the eyeball and eyelid after ocular administration of ${ }^{14} \mathrm{C}-\mathrm{SA} 22465$

\begin{tabular}{|c|c|c|c|c|c|c|c|c|}
\hline Animal number and sex & $1, \mathrm{M}$ & $2, \mathrm{M}$ & $3, \mathrm{M}$ & $4, \mathrm{M}$ & $5, \mathrm{M}$ & $6, \mathrm{M}$ & $7, \mathrm{M}$ & $8, \mathrm{M}$ \\
\hline Tissue Sampling time & \multicolumn{2}{|c|}{1 hour } & \multicolumn{2}{|c|}{4 hours } & \multicolumn{2}{|c|}{8 hours } & \multicolumn{2}{|c|}{24 hours } \\
\hline \multicolumn{9}{|l|}{ Eye } \\
\hline Lens & + & + & + & + & + & + & + & + \\
\hline Cornea & ++ & ++ & ++ & +++ & ++ & ++ & ++ & + \\
\hline Ciliary body $^{1}$ & +++ & +++ & +++ & +++ & +++ & ++ & ++ & ++ \\
\hline Iris & ++ & ++ & ++ & ++ & ++ & ++ & ++ & ++ \\
\hline Pupil & + & + & + & + & + & + & + & + \\
\hline Choroid & ++ & ++ & +++ & +++ & +++ & ++ & ++ & ++ \\
\hline Retina & + & + & +++ & +++ & ++ & ++ & ++ & ++ \\
\hline Sclera & +++ & +++ & +++ & +++ & ++ & ++ & ++ & ++ \\
\hline Optic nerve & ++ & ++ & +++ & +++ & + & + & ++ & ++ \\
\hline Aqueous humor & + & + & + & + & + & + & + & + \\
\hline Vitreous humor & + & + & + & + & + & + & + & + \\
\hline \multicolumn{9}{|l|}{ Eyelids } \\
\hline Meibomian gland & ++ & ++ & ++ & ++ & ++ & ++ & ++ & ++ \\
\hline Meibomian duct & + & + & + & + & 0 & 0 & + & + \\
\hline Connective tissue & ++ & ++ & ++ & ++ & ++ & ++ & ++ & ++ \\
\hline Nictitating membrane & ++ & ++ & +++ & +++ & ++ & ++ & ++ & NS \\
\hline Hair follicles & ++ & ++ & +++ & +++ & +++ & +++ & +++ & +++ \\
\hline Surface contamination & ++++ & ++++ & ++++ & ++++ & ++++ & ++++ & ++++ & ++++ \\
\hline Positive control & \multicolumn{8}{|c|}{ ++++ slides completely blackened } \\
\hline
\end{tabular}

++++, Very high levels of silver grains present; +++, high levels of silver grains present; ++, moderate levels of silver grains present; +, low levels of silver grains present. 0 , Background levels of silver grains; 1 , elevated levels compared with quantitative assessment due to pressure artifacts caused by creasing/folding of the tissue against the nuclear emulsion. NS, Not sectioned.

2.3 hours. Therefore, the meibomian orifice must be separated when determining the actual concentration in meibomian glands; otherwise, the value will be overrepresented.

The drug concentration profiles in palpebral conjunctival epithelium were between the values established for lid margins and meibomian glands. This finding is reasonably interpreted because any solution placed on the surface of the eye becomes significantly diluted in the tear film and eliminated through the tear drainage system. Notably, our results demonstrated that betamethasone concentrations in meibomian glands were approximately 4-fold lower than conjunctival epithelium. Simply given that the tarsal plate lays $400 \mu \mathrm{m}$ deep inside the eyelid, the concentration of a drug is theoretically predicted to show a gradient following its application based on Fick's 2nd law of diffusion. Vehicle and formulation components are now widely accepted to influence the rate and extent of passive chemical absorption through epithelial tissue. The product monograph of Garasone states that betamethasone sodium phosphate is rapidly absorbed into the aqueous compartment via the cornea with an average concentration of 0.48 and $1.76 \mu \mathrm{g} / \mathrm{g}$ of tissue, respectively (Garasone, 2013), indicating that betamethasone exhibits a favorable molecular permeability because of its lipophilic properties. Interestingly, corneal thickness in rabbits is approximately $400 \mu \mathrm{m}(\mathrm{Li}$ et al., 1997), identically matching the distance from the surface of the palpebral conjunctiva to the meibomian duct. Taken together, the coincidence in concentration differences associated with distance suggests that the tarsal plate serves as a tissue barrier similar to the cornea. This hypothesis is consistent with the results for the SA22465 ophthalmic suspension, which demonstrated that the concentrations in the meibomian glands were approximately 100 -fold lower than those in the conjunctival epithelium. Indeed, SA22465 concentrations in the cornea and aqueous humor were 19.9 and $0.1 \mathrm{ng} / \mathrm{g}$ of tissue at peak concentrations, respectively, generating a 200-fold concentration gap (data not shown). However, the identity of the molecule or structure that is biologically responsible for the perceived barrier function is unclear, and further investigations are required to confirm this hypothesis.

Microautoradiography revealed more detailed information of ocular distribution. The highest concentrations of radioactivity in the eyelids were associated with hair follicles. This result was likely due to the migration of formulated material along the hair shaft into the follicles, suggesting that hair follicles provide a route of entry into tissue. Indeed, several recent studies demonstrated that a transfollicular pathway contributes to the permeation of topically applied chemicals, especially hydrophilic agents [logarithm of n-octanol/water partition coefficient $\left(\log K_{\mathrm{o} / \mathrm{w}}\right)<0$ ] (Horita et al., 2014; Mohd et al., 2016). However, the transfollicular contribution should be negligible for SA22465-related material because of the high $\log K_{\mathrm{o} / \mathrm{w}}$ value $(>5$ ), and the anatomic feature that meibomian glands, unlike other sebaceous glands, do not have direct connection with hair follicles. Radioactivity was also present in nictitating membranes, which had direct contact with the formulated material. The presence of radioactivity in connective tissue further supports that the SA22465-related material was absorbed. There is evidence that the nictitating membrane serves as a drug reservoir for topically applied drugs (Mindel et al., 1984). Nictitating membrane is composed of cartilage covered by continuous bulbar and palpebral conjunctiva on its inner and outer surfaces; however, humans do not possess this structure. We therefore speculate that the transconjunctiva is the most plausible route into meibomian gland tissue following topical administration. We also investigated the pharmacokinetic profile of meibomian ducts, in which radioactivity matched background levels. In contrast to hair follicles, each generally associated with one acinus, a single meibomian duct is connected to approximately 15 secretory acini, thereby leading to enhanced lipid release. Continuously secreted meibum may prevent materials from entering the duct unlike hair follicles. Indeed, the terminal part of every gland is surrounded by Riolan's muscle, which assists with the delivery of meibum into the orifice (Knop et al., 2011). Therefore, the meibomian duct may be excluded as a likely entry route of radioactivity into the deeper eyelid.

The transconjunctiva appears to be the most promising route of drug delivery into meibomian glands. Conjunctival drug absorption is generally nonproductive because of conjunctival blood capillaries and lymphatics, which cause significant drug loss into the systemic circulation, thereby lowering ocular bioavailability (Gaudana et al., 2010). Therefore, subconjunctival inserts or film may be ideal formulations and exhibit 
Time after instillation

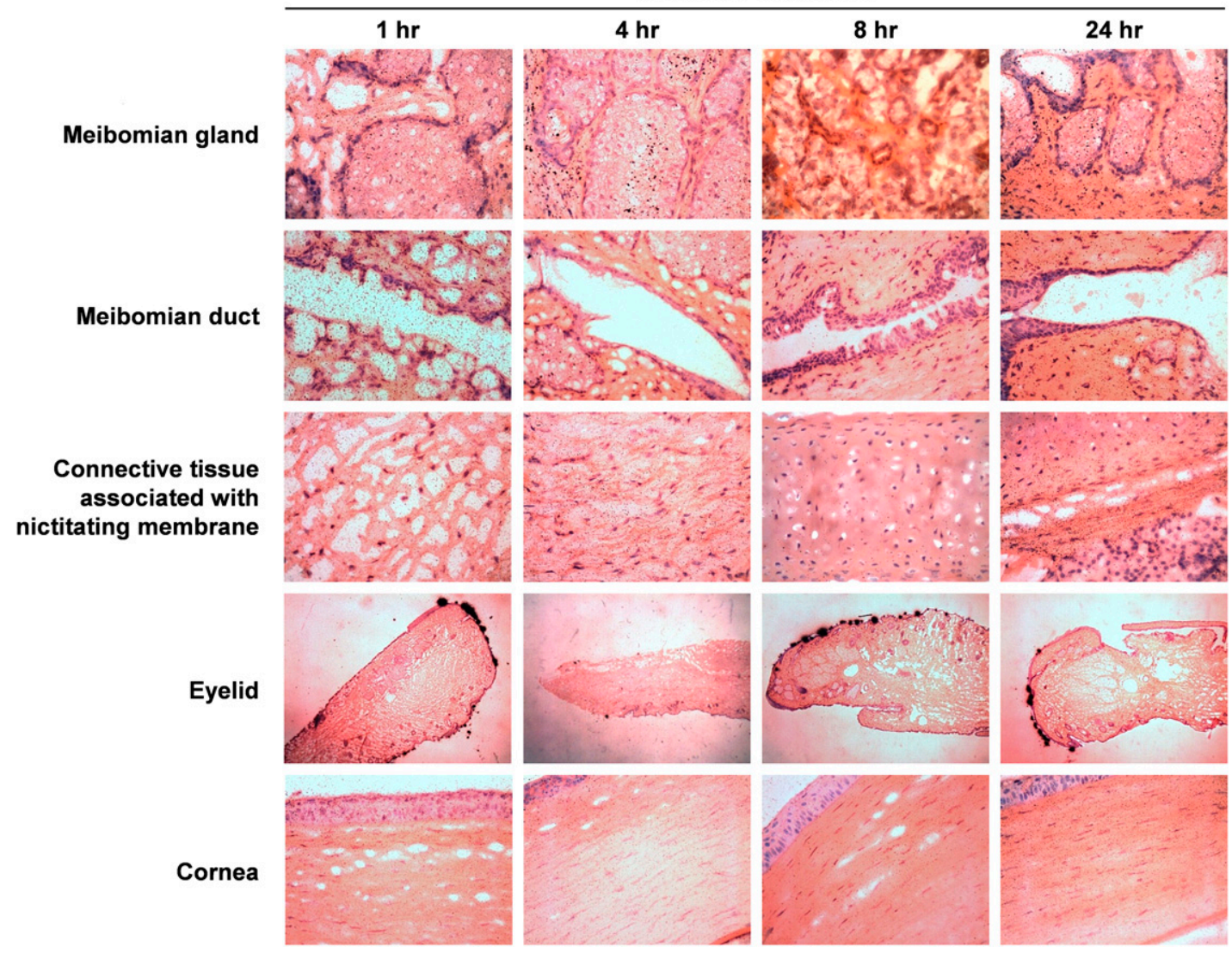

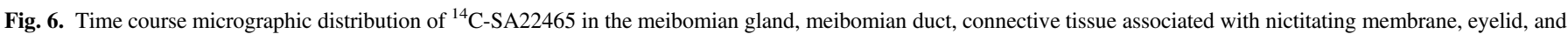
cornea following a single ocular administration. Magnification: $\times 2.5$ for eyelids, $\times 20$ for other tissues.

a comparative advantage over traditional eye drops based on prolonged drug exposure to conjunctival tissue and the prevention of unnecessary drug contamination around the lid margin. A transdermal approach may also be possible if cosmetic issues are resolved because upper eyelids have the thinnest skin compared with other dermal sites of the body (Hwang et al., 2006), suggesting the barrier function may be reduced, especially in the eyelid. However, in addition to the number of cell layers, the size of the corneocytes also contributes to skin permeability (Rougier et al., 1988). Moreover, transdermal eyelid application eliminates tear dilution and reduces the dynamic barrier created by conjunctival blood flow, allowing easier dosing. These positive aspects may produce therapeutic benefits via efficient and controllable drug delivery. However, eyelid drug delivery requires further investigations, especially regarding toxicology and esthetics. Topically applied lipidbased formulations were recently tailored to overcome the challenges of drug delivery to meibomian glands. This approach is theoretically effective because of the similarities in chemical and physical properties between these formulations and meibum, enabling maximum therapeutic effect (Maskin, 2013). That study was completed without pharmacokinetic information; however, it provides valuable insights because pharmacodynamics may be expressed as functions of these concentrations. Having established a new collection method herein, it may prove a useful tool for lead selection and further optimization of formulation. We believe that this pharmacokinetic approach is more suitably carried out in healthy animals with normal eyelid structure and function, rather than for disease models because of individual variation in eyelid damage, resulting in overestimated drug exposure and consequently to inaccurate conclusions. Some disease models may also be adapted to our protocol, depending on how severely the eyelids are swollen or cracking. Overall, our platform may foster a better design for effective meibomian glandtargeting drugs based on pharmacokinetics. To our knowledge, this study is the first report documenting that drug penetration toward the meibomian glands is blocked by a tissue barrier regardless of whether drug is in solution or suspension. How the tarsal plate or peripheral conjunctiva exerts the observed barrier functions is unknown. In vitro studies using freshly isolated human or animal eyelids mounted on a diffusion chamber system are easy to perform using a variety of compounds in concurrent conditions, facilitating a better understanding of the pharmacokinetic characteristics in eyelid tissues.

In summary, our findings indicate that a drug may reach meibomian glands via the transconjunctiva route following ocular administration but with a limited distribution because of a physiologic barrier that restricts drug penetration across the tarsal plate. Autoradiography revealed that the central meibomian duct does not serve as a route of entry for pharmaceutical agents into the meibomian glands.

\section{Acknowledgments}

The authors thank Phil Fernyhough and Brian Whitby (Covance Laboratories Ltd.) for supervision of the autoradiography studies and for valuable comments.

\section{Authorship Contributions}

Participated in research design: Asano and Ueda.

Conducted experiments: Asano.

Contributed new reagents or analytic tools: Asano.

Performed data analysis: Asano.

Wrote or contributed to the writing of the manuscript: Asano, Ueda, and Kawazu. 


\section{References}

Baudouin C, Messmer EM, Aragona P, Geerling G, Akova YA, Benítez-del-Castillo J, Boboridi KG, Merayo-Lloves J, Rolando M, and Labetoulle M (2016) Revisiting the vicious circle of dry eye disease: a focus on the pathophysiology of meibomian gland dysfunction. Br J Ophthalmol 100:300-306.

Bron AJ, Tiffany JM, Gouveia SM, Yokoi N, and Voon LW (2004) Functional aspects of the tear film lipid layer. Exp Eye Res 78:347-360.

Dougherty JM and McCulley JP (1986) Bacterial lipases and chronic blepharitis. Invest Ophthalmol Vis Sci 27:486-491.

Finis D, Hayajneh J, König C, Borrelli M, Schrader S, and Geerling G (2014) Evaluation of an automated thermodynamic treatment (LipiFlow $\left.{ }^{\circledR}\right)$ system for meibomian gland dysfunction: a prospective, randomized, observer-masked trial. Ocul Surf 12:146-154.

Garasone. (2013) Package insert. Merck Canada Inc., Quebec.

Gaudana R, Ananthula HK, Parenky A, and Mitra AK (2010) Ocular drug delivery. AAPS J 12 $348-360$.

Geerling G, Tauber J, Baudouin C, Goto E, Matsumoto Y, O'Brien T, Rolando M, Tsubota K, and Nichols KK (2011) The international workshop on meibomian gland dysfunction: report of the subcommittee on management and treatment of meibomian gland dysfunction. Invest Ophthalmol Vis Sci 52:2050-2064.

Green-Church KB, Butovich I, Willcox M, Borchman D, Paulsen F, Barabino S, and Glasgow BJ (2011) The international workshop on meibomian gland dysfunction: report of the subcommittee on tear film lipids and lipid-protein interactions in health and disease. Invest Ophthalmol Vis Sci 52:1979-1993.

Horita D, Yoshimoto M, Todo H, and Sugibayashi K (2014) Analysis of hair follicle penetration of lidocaine and fluorescein isothiocyanate-dextran $4 \mathrm{kDa}$ using hair follicle-plugging method. Drug Dev Ind Pharm 40:345-351.

Hwang K, Kim DJ, and Hwang SH (2006) Thickness of Korean upper eyelid skin at different levels. J Craniofac Surg 17:54-56.

Jester JV, Parfitt GJ, and Brown DJ (2015) Meibomian gland dysfunction: hyperkeratinization or atrophy? BMC Ophthalmol 15 (Suppl 1):156.

Knop E, Knop N, Millar T, Obata H, and Sullivan DA (2011) The international workshop on meibomian gland dysfunction: report of the subcommittee on anatomy, physiology, and pathophysiology of the meibomian gland. Invest Ophthalmol Vis Sci 52:1938-1978.

Lemp MA and Nichols KK (2009) Blepharitis in the United States 2009: a survey-based perspective on prevalence and treatment. Ocul Surf 7(Suppl 2):S1-S14.

Li HF, Petroll WM, Møller-Pedersen T, Maurer JK, Cavanagh HD, and Jester JV (1997) Epithelia and corneal thickness measurements by in vivo confocal microscopy through focusing (CMTF). Curr Eye Res 16:214-221.

Maroudas A (1976) Transport of solutes through cartilage: permeability to large molecules. J Anat 122:335-347.

Maskin SL (2010) Intraductal meibomian gland probing relieves symptoms of obstructive meibomian gland dysfunction. Cornea 29:1145-1152.
Maskin SL(2013) inventor, MELBJ Holdings, LLC, assignee. Treatment for meibomian gland dysfunction or obstruction. U.S. patent 8,455,016. 2013 Jan 6.

Maskin SL and Tseng SC (1991) Culture of rabbit meibomian gland using collagen gel. Invest Ophthalmol Vis Sci 32:214-223.

Maurice DM (1980) Factors influencing the penetration of topically applied drugs. Int Ophthalmol Clin 20:21-32.

Milz S, Neufang J, Higashiyama I, Putz R, and Benjamin M (2005) An immunohistochemical study of the extracellular matrix of the tarsal plate in the upper eyelid in human beings. J Anat 206:37-45.

Mindel JS, Smith H, Jacobs M, Kharlamb AB, and Friedman AH (1984) Drug reservoirs in topical therapy. Invest Ophthalmol Vis Sci 25:346-350.

Mohd F, Todo H, Yoshimoto M, Yusuf E, and Sugibayashi K (2016) Contribution of the Hai Follicular Pathway to Total Skin Permeation of Topically Applied and Exposed Chemicals. Pharmaceutics 8:32.

Nagataki S and Mishima S (1980) Pharmacokinetics of instilled drugs in the human eye. Int Ophthalmol Clin 20:33-49.

Qiao J and Yan X (2013) Emerging treatment options for meibomian gland dysfunction. Clin Ophthalmol 7:1797-1803.

Rougier A, Lotte C, Corcuff P, and Maibach HI (1988) Relationship between skin permeability and corneocyte size according to anatomic site, age, and sex in man. $J$ Soc Cosmet Chem 39:15-26. Samtani MN, Lohle M, Grant A, Nathanielsz PW, and Jusko WJ (2005) Betamethasone pharmacokinetics after two prodrug formulations in sheep: implications for antenatal corticosteroid use. Drug Metab Dispos 33:1124-1130.

Schaumberg DA, Nichols JJ, Papas EB, Tong L, Uchino M, and Nichols KK (2011) The international workshop on meibomian gland dysfunction: report of the subcommittee on the epidemiology of, and associated risk factors for, MGD. Invest Ophthalmol Vis Sci 52: 1994-2005.

Siak JJK, Tong L, Wong WL, Cajucom-Uy H, Rosman M, Saw SM, and Wong TY (2012) Prevalence and risk factors of meibomian gland dysfunction: the Singapore Malay eye study. Cornea 31:1223-1228.

Viso E, Rodríguez-Ares MT, Abelenda D, Oubiña B, and Gude F (2012) Prevalence of asymptomatic and symptomatic meibomian gland dysfunction in the general population of Spain Invest Ophthalmol Vis Sci 53:2601-2606.

Wladis EJ, Bradley EA, Bilyk JR, Yen MT, and Mawn LA (2016) Oral antibiotics for meibomian gland-related ocular surface disease: a report by the American academy of ophthalmology. Ophthalmology 123:492-496.

Address correspondence to: Nagayoshi Asano, Pharmacology Group, Santen Pharmaceutical Co., Ltd., 8916-16, Takayama-cho, Ikoma-city, Nara, 630-0101, Japan. E-mail: nagayoshi.asano@santen.co.jp 